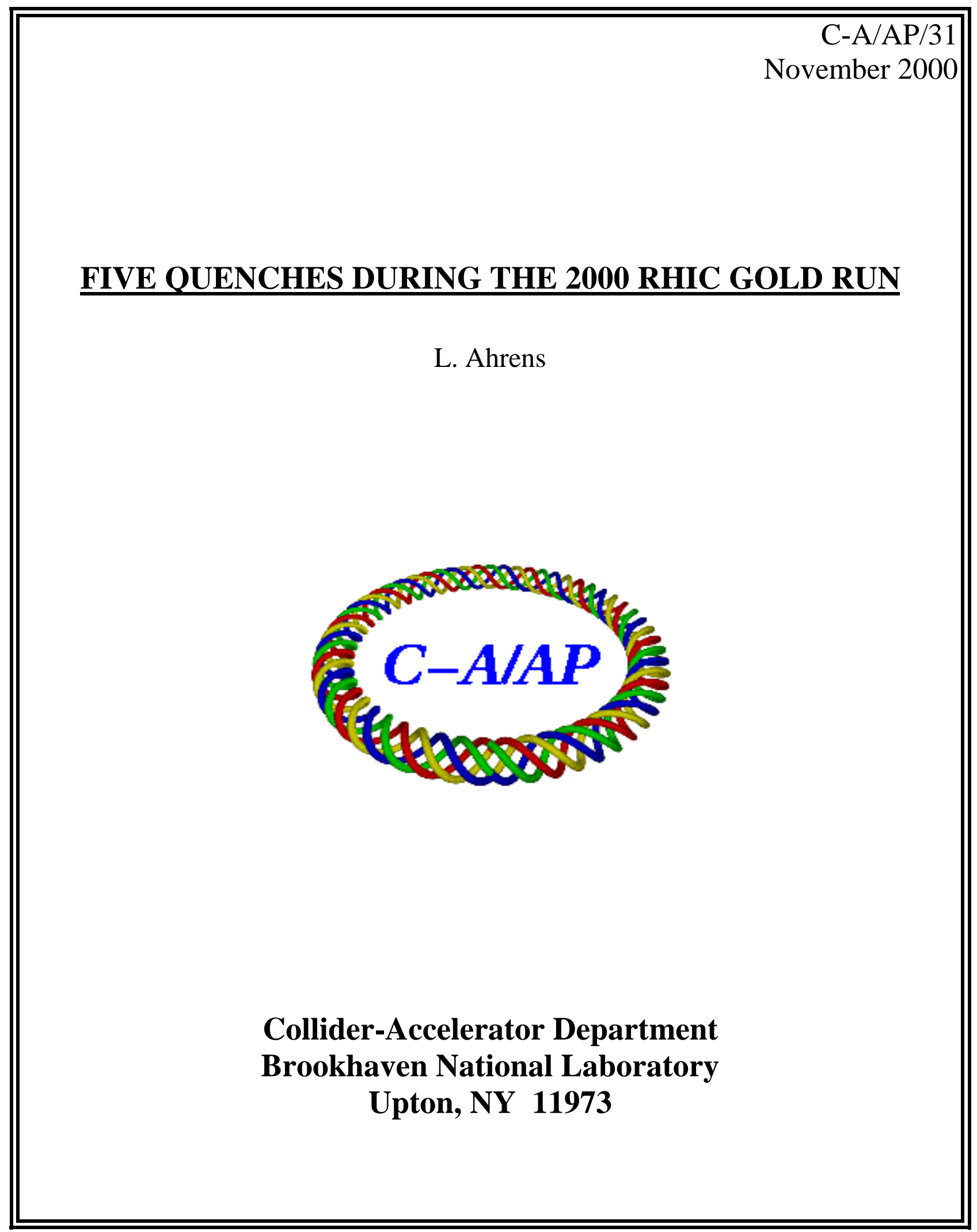


The purpose of this note is to present several pieces of information collected from five real magnet quenches apparently due to beam losses that occurred during August and September 2000. There are at least two motivations. We are extending the "Postmortem" application for RHIC. This application should do this information gathering each time we remove the beam with the dump systems. We are partially answering the question: What data is useful to gather? The data reviewed in this note may also be trying to teach us something about how the machine and the diagnostics respond to these "beam loss and real magnet quench" situations.

A few words (two paragraphs) of caution about the jargon in the following discussion are probably necessary. In RHIC there are three relevant "links": the permit link, the blue quench link, and the yellow quench link. (We are not talking about the event sending links i.e. the RHIC event link, the Blue beam sync link, and the Yellow beam sync link). The permit link must be "up" for there to be beam in either ring. The blue or yellow quench link must be up for there to be magnet current in the associated magnet ring. Each of these links is a closed loop around which a "carrier" propagates. (A given transition of the carrier signal on any one of these three loops makes its round trip in about 30 usec. A beam bunch makes it around the ring in about 13 usec.) The three loops wind through lots of electronic boxes, any one of which can interrupt the associated carrier. This interruption then propagates on around the associated loop. The carrier in that loop has been "dropped". The link has been "pulled" or broken. If one of the quench links is dropped, then the permit link will also be dropped. Beam will be removed and current removed from the ring that has lost its quench link. The other quench link (and the current in that ring of magnets) need not be dropped. If the permit link is dropped, neither quench link need be dropped. The beam will be gone.

We remove the beam from RHIC using the abort kicker and abort absorber system. Now more jargon. If we intentionally remove the beam, e.g. to end a store because we decide that the intensity has dropped too low, then we "dump" (not "abort") the beam. We sent the RHIC event link events: ev-bdump and ev-ydump - which events initiate the triggering of the two kicker systems and the removal of the two beams. Alternatively, if something goes wrong and the permit link is dropped, then the kickers in both rings are automatically triggered. We say that the beam has been "aborted" (not "dumped"). When any of the three link carriers disappears, a monitoring module in $1004 \mathrm{~b}$ recognizes that fact. This module then sends the appropriate events out on the RHIC event link i.e. ev-beamabort for the permit, and ev-bquench or ev-yquench for the quench links.

During August, 2000, the collider was typically filled with 56 bunches in each ring with total intensities of about $1 \times 10^{10}$ ions per ring. Beam energy at store corresponded to a gamma of 70. An issue in predicting trouble for the future is associated with the "margin" present in a superconducting magnet when the scraping beam deposits energy into it. Because of the lower beam intensity, and especially because of the lower current in the magnets at store (the lower beam energy), this margin was significantly larger during the 2000 run than it will be for the next run $\left(5 \times 10^{10}\right.$ ions and a gamma of 100). What does "significantly" mean? Alan Stevens is willing to make a "crude guesstimate" with lots of assumptions, which I mostly do not go into. If we are within $20 \%$ of the quench limit under normal running conditions at gamma $=100$, then compared 
to last run our margin will be down by at least a factor of three due to the higher energy. We will also be down by about another factor of 3 because we will have higher intensity stored beam.

There are five events described below. Two, perhaps three, were identified rather quickly during the run (within a shift or two) as probable real quenches. Two others were predicted to be quenches (a month later) based on loss monitor data. The signature used here to identify a quench has two parts. The first is a unique quench voltage excursion associated with the blue or yellow quench link going down and seen in the standard magnet voltage data collected in association with every quench link pull. This data is so far reserved for traveling experts. It is accessible only in 1004b. Jon Sandberg and George Ganetis have supplied this. The second part of the signature is an associated excursion in the ring loss monitor outputs at nearly the same azimuthal ring location (as that of the magnet with the quench voltage excursion). It is true I believe that there has not been a thorough search over all the run data for additional events - in either category. In fact, one of the results from the evolving Postmortum application will (should) be the ability to do such a search efficiently. There is no mention of the information from the Cryo group - filling "recoolers" etc. here. That information has not been tapped - if it exists - and in a real sense is most relevant.

The following tables contain data relevant to the quenches. Most of this data should be included in Postmortem collections of the future.

Table 1: The Events -time, location, cause

\begin{tabular}{|l|l|l|l|l|l|}
\hline event & date & time & $\begin{array}{l}\text { location } \\
\text { from mag } \\
\text { voltage) }\end{array}$ & $\begin{array}{l}\text { location } \\
\text { (from loss } \\
\text { monitor) }\end{array}$ & $\begin{array}{l}\text { probable associated } \\
\text { “operator" action }\end{array}$ \\
\hline 1 & 19Aug00 & 01.14 .48 & B3QFQ2 & $\begin{array}{l}\text { g3-lm1 } \\
\text { b3-lm0 }\end{array}$ & $\begin{array}{l}\text { "ramp2inj" } \\
\text { "operator" error }\end{array}$ \\
\hline 2 & 27 Aug00 & 23.04 .10 & Y7QFQ3 & (no data) & $\begin{array}{l}\text { corrector saturation } \\
\text { program problem }\end{array}$ \\
\hline 3 & 29 Aug00 & 04.50 .24 & B11QFQ2 & g11-lm1 & $\begin{array}{l}\text { access to ring: no } \\
\text { dump, rf off, PASS }\end{array}$ \\
\hline 4 & 30 Aug00 & 08.41 .51 & Y7QFQ3 & $\begin{array}{l}\text { y7-lm3.1 } \\
\text { y7-lm4 }\end{array}$ & $\begin{array}{l}\text { corrector saturation } \\
\text { program problem }\end{array}$ \\
\hline 5 & 3 Sep00 & 00.15 .47 & B3QFQ3 & b3-lm3 & $\begin{array}{l}\text { (? blue snake } \\
\text { at full power ?) }\end{array}$ \\
\hline
\end{tabular}

Table 2: The Events: rings, intensity, channels

\begin{tabular}{|l|l|l|l|l|l|}
\hline event & $\begin{array}{l}\text { quench link } \\
\text { pulled }\end{array}$ & $\begin{array}{l}\text { intensity } \\
\text { ions }\left(\times 10^{10}\right) \\
\text { blue }\end{array}$ & $\begin{array}{l}\text { intensity } \\
\text { ions }\left(\times 10^{10}\right) \\
\text { yellow }\end{array}$ & $\begin{array}{l}\text { quench } \\
\text { channel }\end{array}$ & $\begin{array}{l}\text { quench/permit } \\
\text { ado }\end{array}$ \\
\hline 1 & blue & 1.00 & 0.75 & 4b-qd1 & 4b-time.A \\
\hline
\end{tabular}




\begin{tabular}{|l|l|l|l|c|c|}
\hline 2 & yellow & 1.40 & 0.80 & $8 \mathrm{~b}-\mathrm{ad} 2$ & $8 \mathrm{~b}-\mathrm{ps} 1$ \\
\hline 3 & blue & 1.90 & 0.00 & $12 \mathrm{a}-\mathrm{ad} 1$ & 12a-ps1.A \\
\hline 4 & yellow & 1.10 & 1.10 & $8 \mathrm{~b}-\mathrm{ad} 2$ & $8 \mathrm{~b}-\mathrm{ps} 1$ \\
\hline 5 & blue & 1.30 & 1.30 & $4 \mathrm{~b}-\mathrm{ad} 1$ & 4b-time.A \\
\hline
\end{tabular}

Table 3: The Events: time stamps

\begin{tabular}{|l|c|c|c|}
\hline event & $\begin{array}{c}\text { 1st quench } \\
\text { timestamp }\end{array}$ & $\begin{array}{c}\text { associated } \\
\text { permit } \\
\text { timestamp }\end{array}$ & $\begin{array}{c}\text { delta in } \\
\text { timestamp }\end{array}$ \\
\hline 1 & 2616639 & 2616670 & +31 \\
\hline 2 & 448358 & 448388 & +30 \\
\hline 3 & 1661216 & 1412085 & -249131 \\
\hline 4 & 129103 & 129133 & +30 \\
\hline 5 & 895558 & 895589 & +31 \\
\hline
\end{tabular}

This last table is a bit cryptic. The numbers listed are from the (archived) "Quench Summary" pet page. Quench time-stamps mark the time when the signal requesting that the quench link be pulled comes in to the permit module (or equivalently ado). In fact, the time listed is the earliest such time stamp looking through all the quench link ado channels listed. The "associated" permit time stamp marks the time when the permit carrier was seen to go away by the same module whose quench time stamp was given - i.e. the first quench link drop listed. When the quench link is pulled, the permit link is also pulled. Then the loss of the permit carrier propagates around the permit link. The module that pulled the permit is necessarily the last to see the permit carrier go away - at its input. Therefore one expects the delay between the two times to be the propagation delay - which is about 30 usec. If this much delay is not seen, then something peculiar is going on. The quench link was not responsible for the permit going down.

Some additional observations on these events:

Event 1:

This event is unique in that the loss monitors associated with it (meaning losses measured before the beam is kicked out of the machine) do not saturate. Apparently the references being sent to the corrector magnets as a result of the "here2inject" command while at store result in an equilibrium orbit distortion that causes beam loss at (and only at) blue 3qf2 for a brief period (less than a second) Then for some reason the beam moves away from this (and any other) aperture. The loss monitors down stream of the quenching magnet (g3-lm1 and b3-lm0) show an increasing signal for about $800 \mathrm{~ms}$, reaching near saturation levels (4000 counts), and then show a reduction consistent with a complete removal of the loss source (see figure 1). The peak in the reported loss occurs about $250 \mathrm{~ms}$ before the blue quench link and the permit link are pulled. If this loss is assumed to cause the quench, it must be true that the time between loss and quench link pull is at least $250 \mathrm{~ms}$. Aborting the beam (removing it from the ring) just at the peak of the 
loss signal would surely have been too late to avoid the quench, but by how much is not clear. The loss monitor reports the beam loss with good fidelity on this $100 \mathrm{~ms}$ time scale. The time interval between the magnet going passed the point of no return and the quench link going down could be longer than $250 \mathrm{~ms}$, if the loss was already sufficient to cause the quench some time before the max loss occurred. Measured losses cross 1000 counts about $500 \mathrm{~ms}$ before the links go down.

It would be instructive to look at the blue intensity over the last second of survival to answer the question how much beam was scraped away to generate this situation. The generic logged current data ( $1 \mathrm{sec}$ sample step) is not sampled frequently enough to give the desired information (say $10 \mathrm{~ms} \mathrm{step).} \mathrm{It} \mathrm{would} \mathrm{be} \mathrm{even} \mathrm{more} \mathrm{instructive} \mathrm{to} \mathrm{watch} \mathrm{how} \mathrm{the} \mathrm{equilibrium}$ orbit moves over this same period.

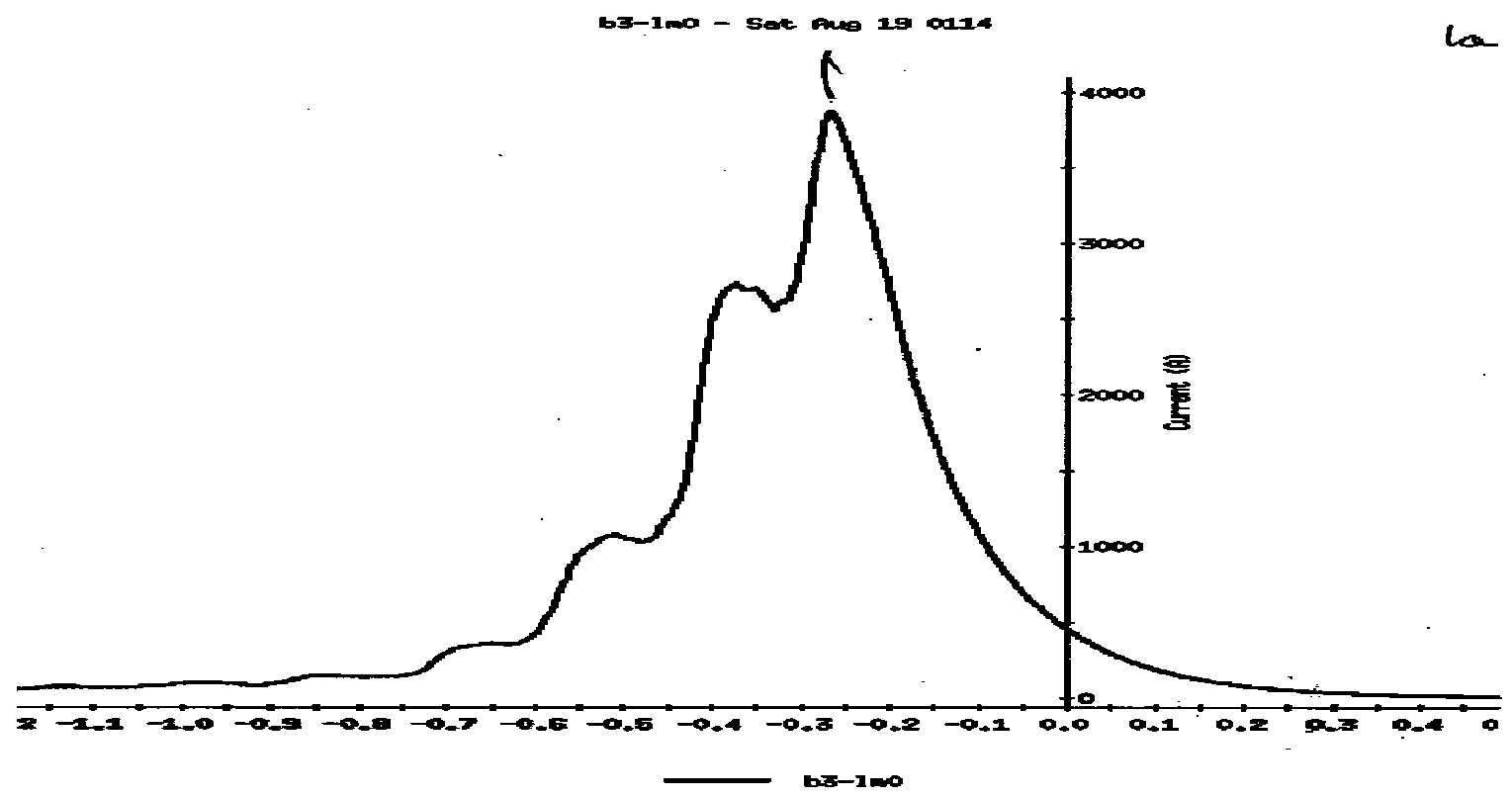

Figure 1 Event 1: Loss Monitor Response Associated with the Quenched Magnet. Time scale $50 \mathrm{~ms} / \mathrm{tick}$, time zero = occurrence of ev-beamabort. 


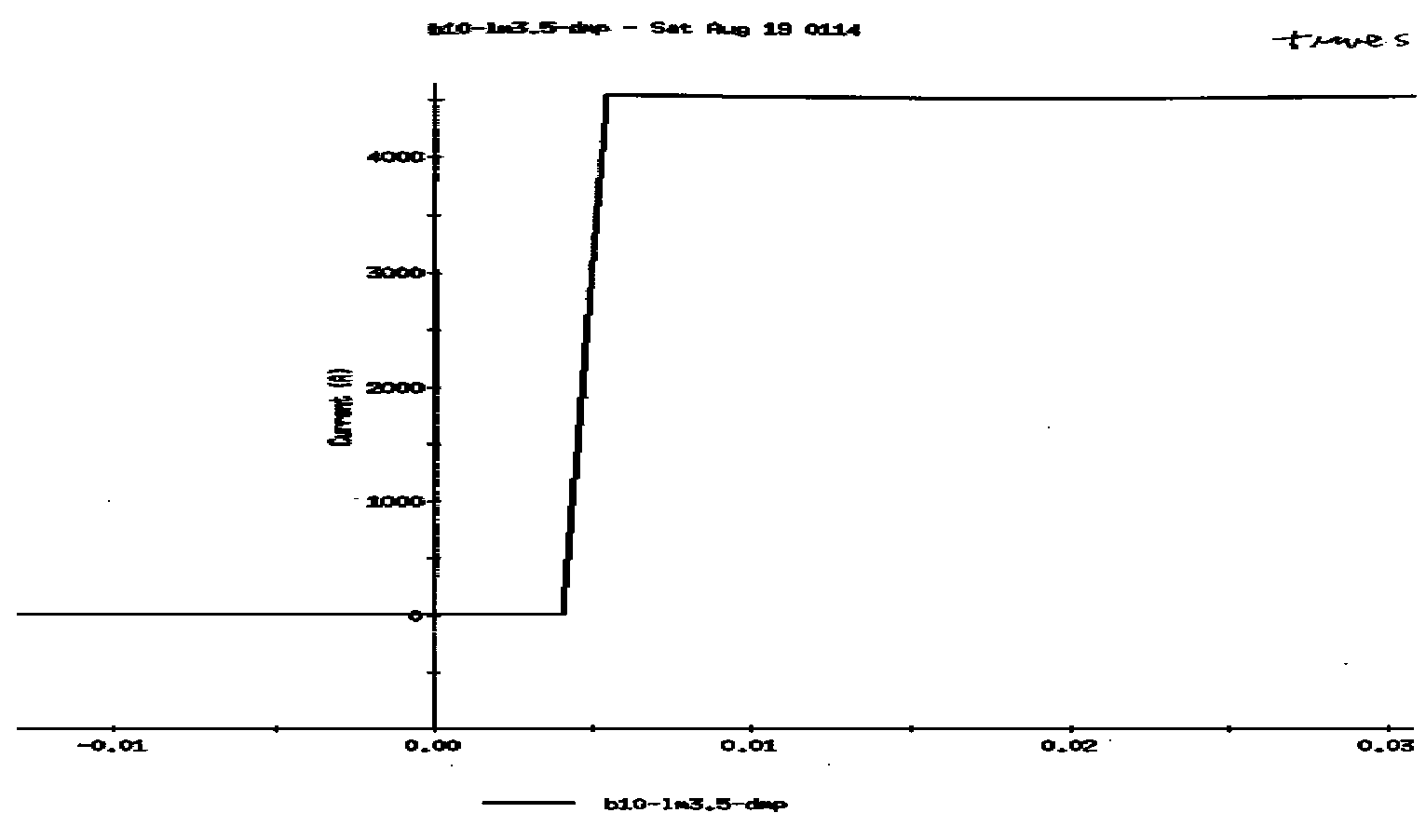

Figure 2 Event 1: Loss Monitor Response at the Dump. The time scale is now $5 \mathrm{~ms} / \mathrm{tick}$ and the monitor saturates in one tick $(1 / 720 \mathrm{sec})$. The time zero is (as always) the occurrence of the RHIC event link event ev-beamabort.

Event 2:

We do not have loss monitor data for this event. It is associated with an interaction point (I.P.) bump scan, and along with event 4 is affectionately referred to as a Fulvia quench. In both cases a bump was applied which pushed some correctors to their maximum currents. Then the command to zero the bump was sent, and misinterpreted by the software as a request to go to full reverse current. (I may have this sequence wrong in detail). At any rate, the bump became unclosed, and so produced oscillations in the equilibrium orbit around the machine. Since we have no loss data for event 2, we can go no further. (See event 4 however).

Event 3:

This is a very different event from the others in this set; with the permit link going down about $250 \mathrm{~ms}$ before the blue quench link drops. The (reconstructed) sequence of events is as follows: Yellow beam was lost during acceleration, blue survived very well into store. The Cryo group requested a ring access. The beam was not dumped. The rf was turned off. An access into the ring was initiated. This required the PASS system to pull the permit, which it did, and the beam was then properly aborted. A quadrupole magnet (b11qf2) just before the 12 o'clock i.p. quenched. The betatron phase advance from the abort kicker to this horizontal beta max quadrupole is about 4.75 - which is to say the betatron oscillation initiated by the kicker is at a maximum at this quadrupole. The betatron function here is significantly larger than anywhere 
upstream back to the kick so the physical excursion of the beam is at maximum. We speculate that the rf being off, (and hence the beam debunched), was the important ingredient to the occurrence of the quench. There was a lot of beam in blue - nearly $2 \times 10^{10}$ ions - (and no beam in yellow). If this conjecture is correct, it is the piece of beam passing through the abort kicker magnets just after the pulse starts to rise but before the kick is sufficient to hit the dump absorber that quenches b11qf2. A crude calculation gives a kick range from .5 to .7 milliradians as targeting the quad. Since the kickers go from 0 to 1.6 milliradians roughly linearly in about 1 usec, the fraction of the debunched beam falling in this kick range is $125 \mathrm{~ns}$ out of $12.8 \mathrm{usec}$, about $1 \%$ of the total, or $2 \times 10^{8}$ ions. If we believe the early on discussion about quench margin reduction next year, we will quench with less than $1 \times 10^{8}$ ions and $.1 \%$ of the total beam in the abort gap. The loss occurs in a single turn. No system can protect the magnet once the beam is debunched.

In this case the measurement of the time between (energy injected) to (quench link pulled) is unambiguous and the answer ( $250 \mathrm{usec}$ ) may help clarify the discussion associated with event 1 . If the delay (quench energy deposition to quench link pull) is about equal everywhere, then the fact that for event 1 the energy deposition (losses) stopped $250 \mathrm{~ms}$ before the quench link was pulled is either coincidence, or perhaps the quenching of the magnet was involved in "correcting" the initial distortion of the equilibrium orbit. (?)

The description of event 3 given here would suggest that it is necessary, if we want to prevent this sort of quench, to insist that the loss of all the rf cavities require that the permit link be pulled. This event also points to a question investigated during the design period, namely: at design energy and intensity how much beam is tolerable in the abort gap buckets?

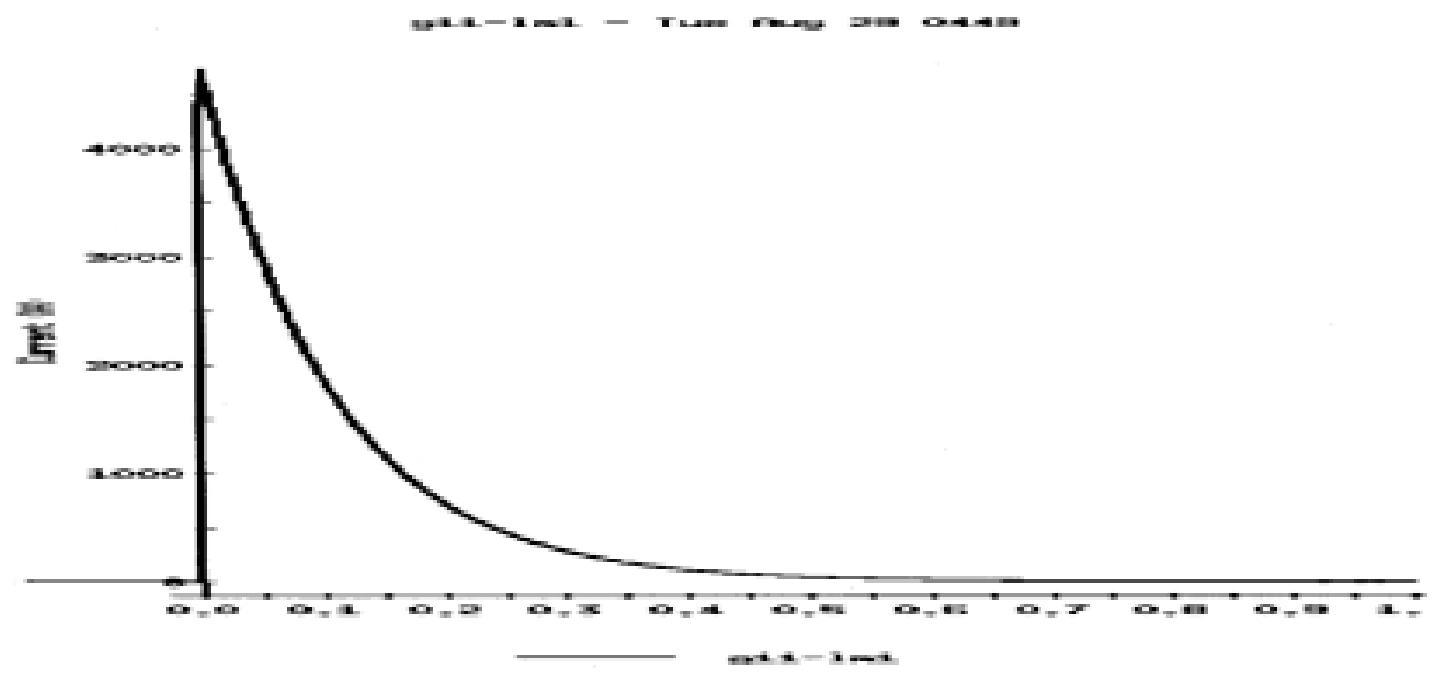

Figure 3 Event 3: Response from loss monitor g11-lm1, located just upstream of the 12 O'clock i.p.

Figure 3 shows the typical time response for monitors in the system to an instantaneous beam loss. (see e.g. the end of figure 1). The signal is flat at near zero until time zero when the beam is aborted. As noted in the text, the blue quench link was pulled at about .25 (sec) on the xaxis - long after the beam was aborted. 


\section{Event 4:}

This is apparently a replay of Event 2 . Now we have the response from the loss monitors. The limiting aperture is y7qf3. There are four special loss monitors downstream of this spot, which are probably more sensitive than the standard loss monitors. The special monitors saturate about $400 \mathrm{~ms}$ before the quench link is pulled. The conventional loss monitor just beside this quadrupole crosses 2000 counts about $400 \mathrm{~ms}$ before the link is pulled, and crosses 4000 counts about $250 \mathrm{~ms}$ before the link goes. This monitor just touches the saturation limit. Equilibrium orbit and intensity information over the last second would be valuable.
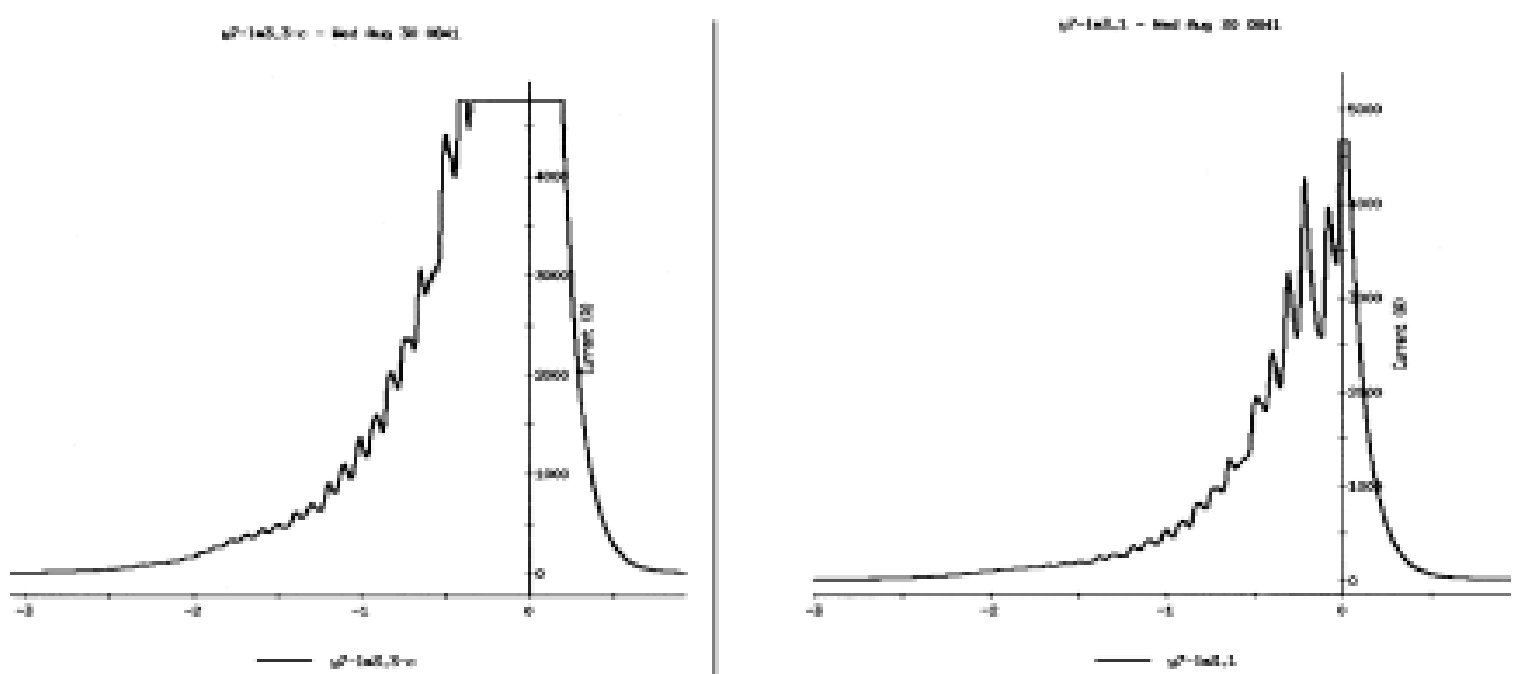

Figure 4 Event 4: Response from Two Loss Monitors near the Quenching Magnet. The monitor on the left is one of a set of nonstandard monitors between q3 and q4. The monitor on the right is a standard monitor - i.e. a monitor with similar geometry relative to any particle spray associated with beam scraping exists for each i.p. The time scale here is $500 \mathrm{~ms} / \mathrm{tick}$.

\section{Event 5:}

There is not a clear explanation for this event in the logbook. Some circumstantial evidence is available. A 30 minute test of the blue snake magnet at full current was initiated 30 minutes before the quench occurred. Perhaps the snake quenched at the end of the high current test and as a result caused a distortion to the equilibrium orbit causing the blue beam to find an aperture in sector 3 ?

Quadrupole b3qf3 quenched. Loss monitor b31m3 went hard into saturation about 400 $\mathrm{ms}$ before the permit was pulled. Some adjacent monitors showed somewhat less enthusiastic, but well correlated, losses, with some additional structure. Clearly again some postmortem data about the equilibrium orbit and beam intensity during the final seconds would be valuable. 

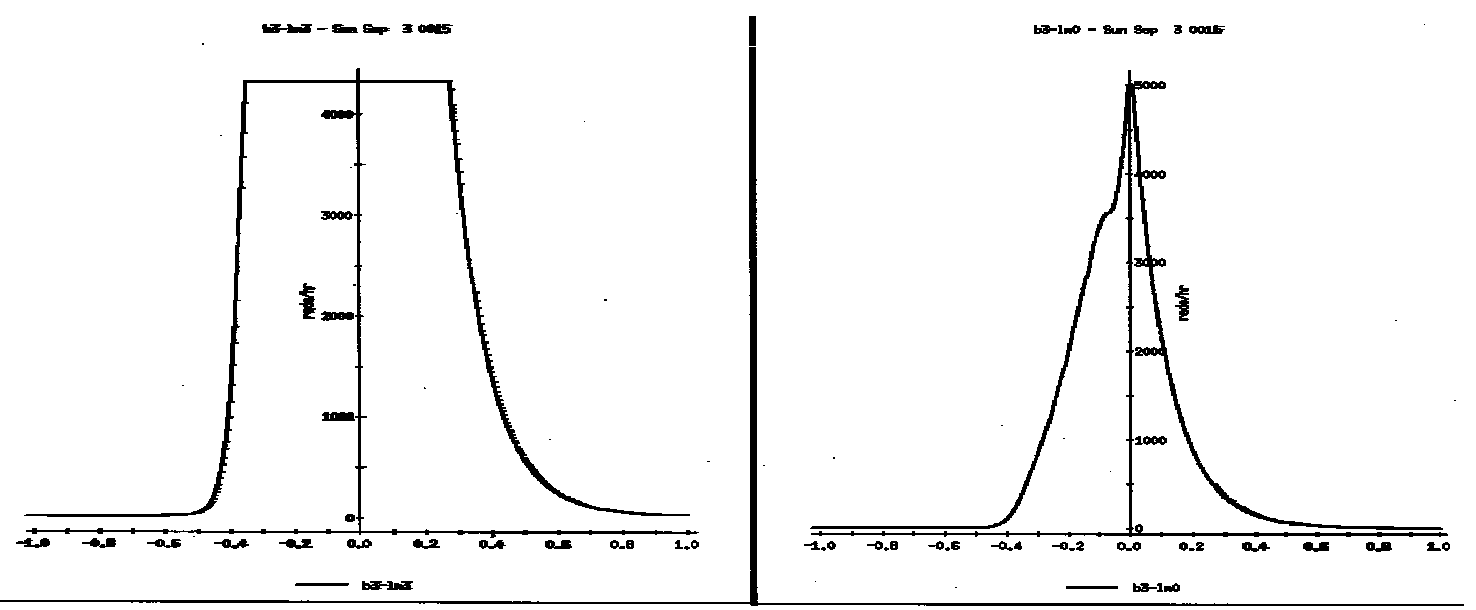

Figure 5 Event 5: The two most enthusiastic loss monitors.

The monitor on the left in figure 5 clearly saturates earlier than $250 \mathrm{~ms}$ before the quench link pull. Apparently this monitor sees losses more effectively than does the magnet about to quench. The details of time structure in the right monitor - i.e. the second hit - is not explained.
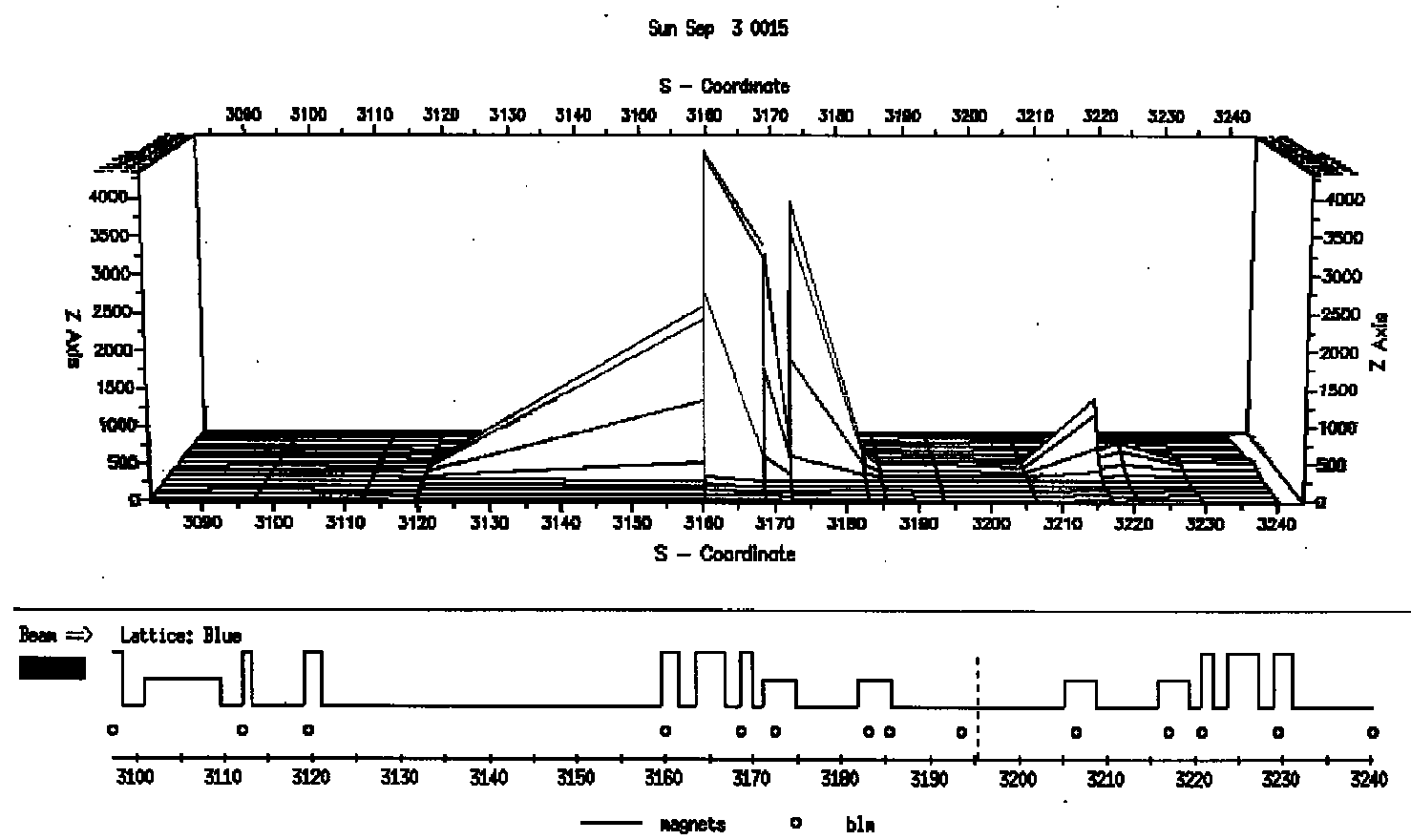

Figure 6 Event 5: Another view of losses associated with event 5. This "mountain range" display shows the loss monitor responses mentioned in the text in the blue ring before I.P.4 (at $3195 \mathrm{~m}$ ). Loss monitor b31m 3 is at $3160 \mathrm{~m}$. Time marches into the picture, with an averaged trace every 10 $\mathrm{ms}$ in this picture.

Some additional discussion:

Decisions will necessarily be taken as to where to "set the bar" choosing between suffering beam aborts as a result of false quench link drops and extremely infrequent real magnet 
quenches or suffering less frequent beam aborts associated with quenched magnets. The latter carry some not well-quantified potential for damaging magnets. Knowing what has happened during real quenches enlightens these decisions. Up till now (i.e. during the beam run) we have not learned experimentally about the damage danger. We do know a bit about the possibility for using the loss monitors to avoid the problem.

All five of these quenches are in I.P. triplet quadrupoles. If we are going to include only a subset of loss monitors in the abort threshold system, monitors located at these locations are at the top of the list. (Sounds like something Waldo has been saying). For the year 2000 running conditions, a threshold setting of 2000 counts may well have cut of the losses soon enough to have prevented four of these quenches. With higher beam energy (hence higher magnet current and magnet fields and energy per ion) the quench threshold must necessarily go down (perhaps to $1 / 3$ of this). What we need to know is how far down the threshold can be lowered without getting into loss monitor noise. At least in the monitor outputs studied here, 2000 counts is well above the noise floor.

Some valuable signals not available last year, and mentioned above include above all a clear "flag" indicating when a loss of the permit is due to a real magnet quench. A good candidate for this exists in data available locally in 1004b. Information from the Cryo group may also exist, perhaps even in MCR. The updating of this information occurs perhaps only at several minute intervals; but that is not a stopper. Other needed signals include: a record of the behavior of the closed orbit over the last few seconds before an abort, and a record of the beam intensity over the same period. And we will have to revisit the rules for required inputs to the permit link.

The author appreciates many discussions with Alan Stevens about all aspects of this business; and the ongoing cooperation with Mei Bai who speaks for the loss monitor system; Jon Laster, who speaks for the Postmortem software; Mike Iarocci, speaking for Cryo (and looking into possible quench information); and both George Ganetis and Jon Sandberg, who speak for the power supplies and the quench links. 